\title{
Histopathological Characterization of a Syngeneic Orthotopic Murine Bladder Cancer Model
}

\author{
Daher C. Chade, Priscila M. Andrade, Ricardo C. Borra, Katia R. Leite, Enrico Andrade, \\ Fabiola E. Villanova, Miguel Srougi
}

Laboratory of Medical Investigation (DCC, PMA, KRL, EA, FEV, MS), Division of Urology, University of Sao Paulo Medical School, and Bioodontology (RCB), Ibirapuera University, Sao Paulo, Brazil

\begin{abstract}
Purpose: We developed and characterized by histopathology and immunohistochemistry a syngeneic murine bladder tumor model derived from the MB49 tumor cell line.

Materials and Methods: Bladder tumor implantation was achieved by intravesical instillation of $5 \times 10^{5}$ MB49 tumor cells in $\mathrm{C} 57 \mathrm{BL} / 6$ mice. A chemical lesion of the bladder was performed in order to promote intravesical tumor implantation. The bladder wall lesion was accomplished by transurethral instillation of silver nitrate $\left(\mathrm{AgNO}_{3}\right)$. After 15 days, the animals were sacrificed, examined macroscopically for intravesical tumor and bladder weight. Histology and immunohistochemistry were performed using cytokeratin 7 (CK7), carcinoembrionic antigen (Dako-CEA), p53 and c-erbB2 oncoprotein (Her2/neu). Results: Twenty-nine out of 30 animals (96.7\%) developed intravesical tumors in a 15-day period. Macroscopically, the mean bladder weight was $0.196 \mathrm{~g}(0.069-0.538 \mathrm{~g}), 10$ to 15 times the normal bladder weight. The immunohistochemical analysis showed significant membrane expression of CEA and CK7: a similar finding for human urothelial cancer. We also characterized absence of expression of p53 and anti-Her2/neu in the murine model.

Conclusions: High tumor take rates were achieved by using the chemical induction of the bladder tumor. Although electric cauterization is widely described in the literature for syngeneic orthotopic animal models, the technique described in this study represents an alternative for intravesical bladder tumor implantation. Moreover, the histopathology and immunohistochemical analysis of the murine bladder tumor model derived from the MB49 cell line showed a resemblance to human infiltrating urothelial carcinoma, allowing clinical inference from experimental immunotherapy testing.
\end{abstract}

Key words: bladder cancer; intravesical instillation; tumor cell line; mice/c57bl; experimental neoplasm Int Braz, J Urol. 2008; 34: 220-9

\section{INTRODUCTION}

Animal models provide a system for understanding basic biological questions. With animal models, adequate control of experimental design is possible so that rigorous experiments can be performed to test various hypotheses. In the case of testing therapeutic mechanisms, it is important to select a model that is most analogous to the clinical setting so that observations can be readily transferred to clinical studies for validation (1).

A murine bladder tumor model may offer some of these characteristics, while having many controlled variables under laboratory conditions (2). This permits inference from experimental data, which can be helpful for clinical purposes. The murine bladder 
tumor models may be a xenograft model (in immunodeficient mice) (3), a chemically induced bladder cancer model (4), or a syngeneic animal model (5).

The use of immunodeficient mice in bladder cancer research has allowed the implantation of human carcinomas in an animal model. In addition, it has demonstrated the importance of the immune system, in particular as regards $\mathrm{T}$ lymphocytes, in anti-tumor activity (6).

The chemically induced models were achieved by administering carcinogens such as $\mathrm{N}$ [4-(5-nitro-2-furyl)-2-thiazolyl] formamide (FANFT) in $\mathrm{C} 3 \mathrm{H} / \mathrm{He}$ mice (7) or 7,12-dimethylbenzanthracene in mice strain C57BL/6 (8). Although adequate for immunotherapeutic testing, a long period of time was required for carcinogen-induced bladder tumor growth.

The syngeneic animal models were developed with the objective of improving immunotherapeutic studies (9). It is characterized by the transplantation of carcinogen-induced bladder cancer into syngeneic immunocompetent mice $(5,10)$. This murine bladder tumor model has been considered appropriate for this purpose, as it permits the possibility of mimicking intravesical conditions. Moreover, research can be improved by testing local tumor response to drugs in an immunocompetent host (11). Implantation of syngeneic tumor cells can be made subcutaneously (heterotopic model) or by intravesical instillation (orthotopic model), in the anatomical site.

In this study, our aim was to characterize the syngeneic orthotopic murine bladder cancer model derived from the MB49 tumor cell line by histopathology and immunohistochemistry. We focused on the urothelial histogenesis of the murine tumor in order to demonstrate its similarities to the human bladder tumor. Therefore, our findings may support its use as an useful experimental bladder tumor model for drug testing and new immunotherapeutic alternatives. In addition, we demonstrate the feasibility of the implantation of the tumor cell line MB49 by the chemical lesion of the bladder using silver nitrate, as described previously by Luo et al. (12). Silver nitrate was chosen for this purpose due to its controlled and stable characteristics. Similar effects may be achieved using ethanol and poly-L-lysine, but this was not tested in this series.
The markers used for immunohistochemistry testing were cytokeratin 7 , carcinoembrionic antigen, p53 and c-erbB2 oncoprotein, all commonly used for evaluating human bladder tumor.

\section{MATERIALS AND METHODS}

Animals - Eight- to 10-week-old female C57BL/6 mice, weighing 15-20g, were provided by the Bioterism Center of the university and maintained at our animal care facility for 1 week prior to use. The mice were housed five per cage in a limited access area at a controlled room temperature, with food and water ad libitum. The experiments were approved by the institution's Ethics Board Council.

Preparation of tumor cells - Syngeneic bladder tumor cell line MB49 was kindly provided by Dr. Yi Lou (University of Iowa, USA). The cells were maintained in vitro culture (DMEM, $10 \% \mathrm{FBS}$, $1 \%$ penicillin/streptomycin, at $37^{\circ} \mathrm{C}$ and $5 \% \mathrm{CO}_{2}$ ). Tumor cells were harvested by trypsinization and suspended in DMEM without L-glutamine, FBS, and antibiotics. Viability was determined by trypan blue exclusion and only tumor cell suspensions with more than $90 \%$ viable cells were used for tumor implantation.

Intravesical tumor implantation - Mice were anesthetized by the intraperitoneal administration of ketamine/xylazine solution at a dose of $0.1 \mathrm{~mL} / 10 \mathrm{~g}$ body weight. Subsequently, a 24-gauge Teflon i.v. catheter $\left(\mathrm{Nipro}^{\mathrm{R}}\right)$ was inserted through the urethra into the bladder using an inert lubricant (sterile contact gel) (Figure-1). Then, in order to prepare the bladder for tumor implantation, a brief acid exposure, followed by alkaline neutralization, promoted a chemical lesion on the bladder wall, performed by intravesical instillation of $8 \mu \mathrm{l} 1 \mathrm{M}$ silver nitrate $\left(\mathrm{AgNO}_{3}\right)$. This promoted an adequate and controlled diffuse bladder wall lesion. After 10 seconds, the content was washed out by transurethral infusion of phosphate-buffered saline. The first catheter was removed and a new 24-gauge catheter was inserted in the urethra for intravesical instillation of MB49 cells. A cell suspension of MB49 tumor cells $\left(5 \times 10^{5}\right.$ cells in $0.1 \mathrm{~mL} 50 \%$ normal mouse serum) was instilled and retained for 2 hours by stitches. 


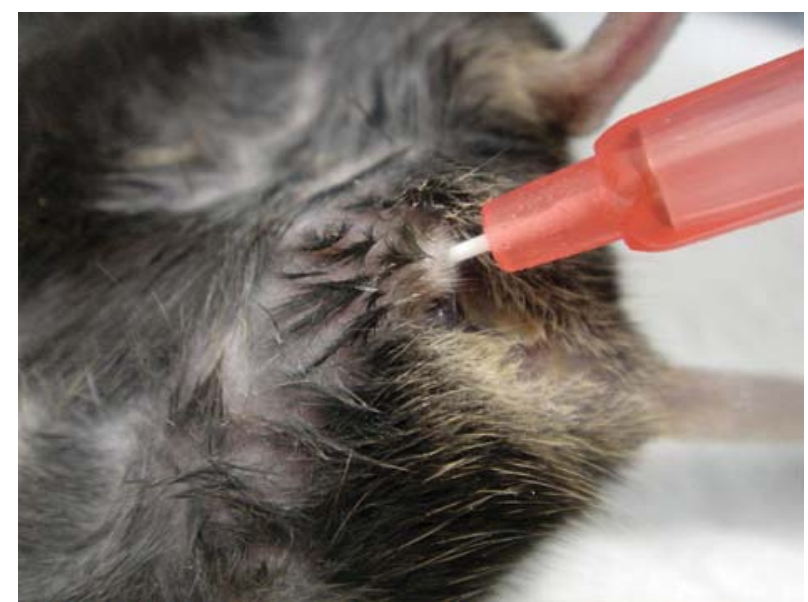

Figure 1-A 24-gauge catheter inserted in the urethra for intravesical instillation (female C57BL/6 mice).

The mice were evaluated on a daily basis for viability and gross hematuria. After 15 days, the animals were sacrificed by $\mathrm{CO}_{2}$ inhalation, examined macroscopically for intravesical tumor and individually verified the bladder weight.

Histology and immunohistochemistry: After gross examination, the bladders were fixed in buffered formalin $10 \%$, routinely processed and paraffin included and stained by hematoxylin and eosin . Immunohistochemistry was performed to characterize the immunophenotype and the antibodies used were cytokeratin 7 (CK7 OV-TL 12/30, 1:100), carcinoembrionic antigen (Dako-CEA, II-7 1:200), p53 (DO7, 1:100) and c-erbB2 oncoprotein (Her2/neu, 1:100), all produced by Dakocytomation, Glostrup, Denmark. Three-micrometer sections from the paraffin block containing tumor were placed on adhesive-coated slides. In a heat antigen retrieval process the slides were placed in a citrate buffer ( $1 \mathrm{mM}, \mathrm{pH} 6.0)$ and heated for $30 \mathrm{~min}$. in the steamer. The slides were incubated overnight at $4^{\circ} \mathrm{C}$ with the above antibodies. Labelled Streptavidin Biotin (LSAB; Dako Cytomation, CA, ) at first biotinylated link universal for $35 \mathrm{~min}$ at room temperature, then the slides were rinsed with Tris-buffer for $5 \mathrm{~min}$., incubated for a further $35 \mathrm{~min}$. with streptavidin-HRP. The slides were rinsed in tap water for $5 \mathrm{~min}$. Color was developed by incubating the slides in $0.06 \%$ diaminobenzidine in PBS for 15 minutes, and the slides were rinsed in Tris-buffer and tap water, counterstained with Harris hematoxylin, dehydrated, cover slipped, and reviewed under light microscope. Tissue sections of a bladder urothelial carcinoma known to express p53 and Her2-neu, as well as pulmonary adenocarcinoma positive for cytokeratin 7 and CEA were used as positive controls. For each case a negative control was applied by following all steps of IHC except for replacement of the primary antibody by PBS.

\section{RESULTS}

All the animals survived the surgical intervention, no transmural bladder injury or bladder perforation was observed. All animals, after 7 days, presented evident gross hematuria (Figure-2), that persisted until the sacrifice on day 15 . There was no obstruction of the urinary flow, except for the gross and intense hematuria. Considering all the animals (30) that received intravesical instillation of MB49

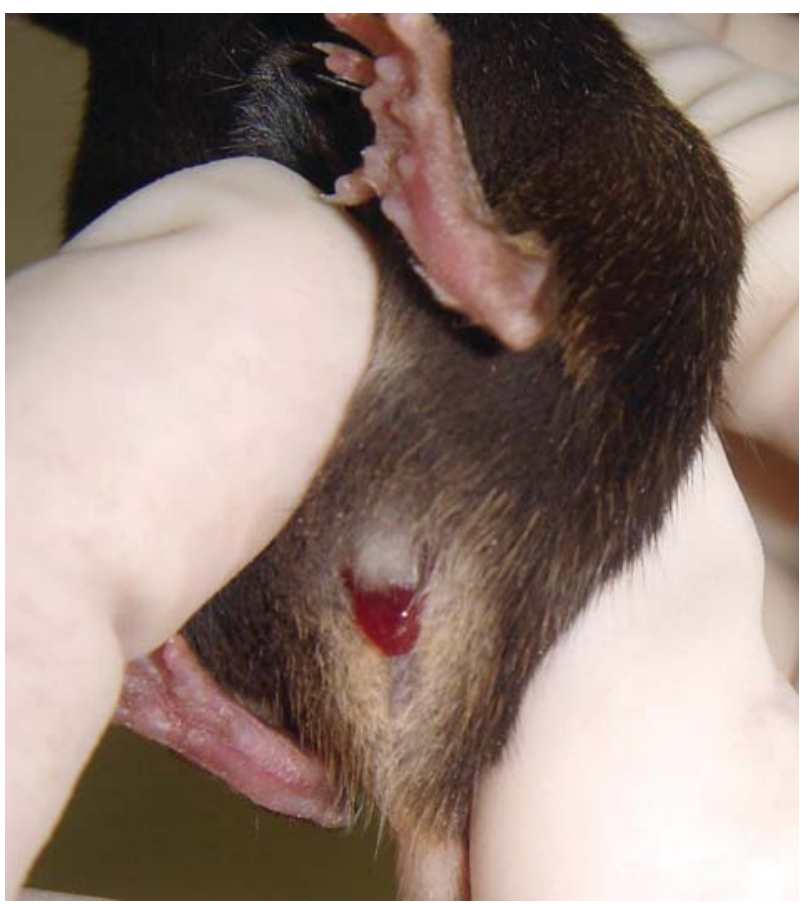

Figure 2-Gross hematuria in a mouse, 7 days after the intravesical instillation of MB49 cells. 
cells, $29(96.7 \%)$ developed intravesical tumors after the 15-day period. At that time there was massive growth of a solid tumor inside the bladder.

Macroscopically the mean bladder weight was $0.196 \mathrm{~g}(0.069-0.538 \mathrm{~g})$ in the tumor group, while a C57BL $/ 6$ mouse bladder weighs between $0.010 \mathrm{~g}$ to $0.015 \mathrm{~g}$ approximately. The tumor was represented by an usually solitary solid mass, growing inside the bladder, deeply red, soft and extremely bloody. There were areas of necrosis and superficial ulceration. Microscopically, there was a high grade urothelial solid carcinoma composed of proliferation of large, cubic cells, arranged in solid nests, with round, hypercromatic nuclei and one or more nucleoli. Scattered giant, bizarre cells were seen in the tumor. The mitosis rate was high (50/HPF). Superficial ulceration and foci of necrosis were identified. In all cases the tumor was invasive through the bladder wall, reaching the muscularis propria. No vascular invasion or perineural infiltration was seen. The transition to normal urothelium was evident, and no in situ carcinoma was identified (Figure-3).

The immunohistochemical analysis showed strong, membrane expression of CEA and CK7: a similar profile that we commonly see for urothelial cancer that affects humans. Although p53 expression is very common in urothelial cancer, due to p53 mutation, in the murine model it does not seem to be a part of the carcinogenesis since we were unable to detect p53 staining. Also, we used antibody anti-Her2/neu, an oncogene super expressed in 30 to $60 \%$ of human urothelial carcinomas. There was no Her2/neu expression in the cases we have evaluated. The molecular evaluation of those two abnormalities among others should be the subject of our next study.

\section{COMMENTS}

As demonstrated above, the histological and immunohistochemical characteristics described in the murine bladder tumor model derived from the MB49 cell line mimics one of the main aspects of the human infiltrating urothelial carcinoma, which is defined as a urothelial tumor that invades beyond the lamina propria. By producing a pathologically similar cancer to the human urothelial carcinoma, evidence from experimental research may become closer to data from human testing.

Considering the immunoregulatory mechanisms that participate in bladder tumor-host interaction (12), a wide field of research is open for investigation. Consequently, adequate local and systemic experimental environment model is required, allowing a significant conclusion to be obtained for further studies.

A murine bladder tumor model may offer some of these characteristics, while having many controlled variables under laboratory conditions. These factors permit inference from experimental data, which can be helpful for clinical purposes, specially considering safety issues.

Despite the technical difficulty of orthotopic tumor implantation, improvements have been made to avoid the variability of tumor cell adhesion to the bladder wall (5). Soloway et al. simulated a transurethral fulguration by cauterizing the murine bladder wall, creating conditions for tumor cells implantation (10). The development of this technical modification enhanced the model's applicability by transforming the orthotopic syngeneic bladder tumor model into a reliable tool for investigating tumor growth mechanisms and intravesical drug testing. Furthermore, the model closely resembles the clinical situation, making it very suitable for bladder cancer research.

The use of immunohistochemistry for accurate diagnosis of cancer has long been demonstrated (13). Determination of cytokeratins, in this situation, may be helpful in tumors poorly differentiated and for identifying the primary site of metastatic carcinomas (14). Moreover, IHC provides information on tumor progression, giving p53 expression among other markers, a significant prognostic value $(15,16)$.

The murine bladder tumor model not only creates adequate conditions for understanding tumor adhesion, proliferation and invasiveness, but also allows the development of a cancer with many similar histopathological characteristics to the human urothelial carcinoma, making it a valuable tool for anti-tumor drug testing, based on immune, viral or gene therapy $(9,17,18)$. 

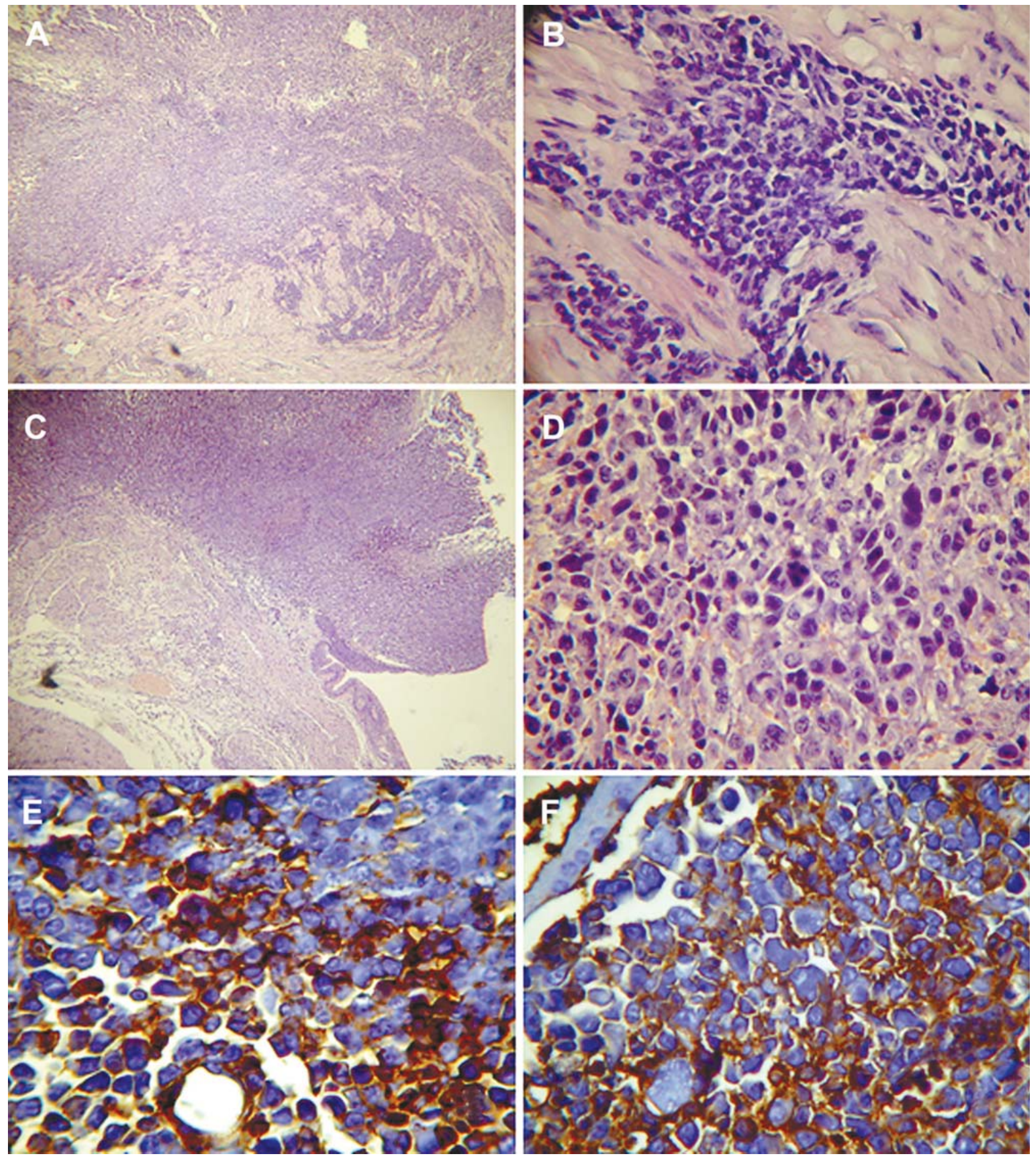

Figure 3 - A) High grade urothelial carcinoma, extensively infiltrative in the muscularis propria, HE X40 and B) X400. C) High grade urothelial carcinoma characterized by a exophytic growth through the vesical lumen, diffusely infiltrative in the lamina propria. Note the clear distinction between normal urothelium and tumor, HE, X40. D) High grade carcinoma characterized by large cubic cells, with intense nuclei polymorphism and high mitotic activity, HE, X400. E) Membrane immune expression of cytokeratin 7, X400, and F) $C E A, X 400$. 


\section{CONCLUSIONS}

The tumor implantation procedures described herein provide a reproducible experimental bladder cancer model. The orthotopic murine model has an important role improving our knowledge of therapeutic mechanisms of superficial bladder cancer in the proper anatomical site.

In conclusion, the histopathology and immunohistochemical profile of the murine bladder tumor model derived from the MB49 cell line resembles the human infiltrating urothelial carcinoma, allowing us to make inferences about its behavior and response to different treatment regimes.

\section{ACKNOWLEDGEMENT}

State of Sao Paulo Research Foundation (FAPESP) provided financial support.

\section{CONFLICT OF INTEREST}

None declared.

\section{REFERENCES}

1. Böhle A, Brandau S: Immune mechanisms in bacillus Calmette-Guerin immunotherapy for superficial bladder cancer. J Urol. 2003; 170: 964-9.

2. Ratliff TL: Role of animal models in understanding intravesical therapy with bacille Calmette-Guérin. Clin Infect Dis. 2000; 31 Suppl 3: S106-8.

3. Hansson Y, Paulie S, Ben-Aïssa H, Rudberg U, Karlsson A, Perlmann P: Radioimmunolocalisation of bladder tumors xenotransplanted in nude mice. Anticancer Res. 1988; 8: 435-41.

4. Soloway MS: Single and combination chemotherapy for primary murine bladder cancer. Cancer. 1975; 36: 333-40.

5. Günther JH, Jurczok A, Wulf T, Brandau S, Deinert I, Jocham D, et al.: Optimizing syngeneic orthotopic murine bladder cancer (MB49). Cancer Res. 1999; 59: 2834-7.

6. Abe T, Tada M, Shinohara N, Okada F, Itoh T, Hamada J, Harabayashi T, Chen Q, Moriuchi T, Nonomura $\mathrm{K}$ : Establishment and characterization of human urothelial cancer xenografts in severe combined immunodeficient mice. Int J Urol. 2006; 13: 47-57.

7. Mickey DD, Mickey GH, Murphy WM, Niell HB, Soloway MS: In vitro characterization of four N-[4(5-nitro-2-furyl)-2-thiazolyl] formamide (FANFT) induced mouse bladder tumors. J Urol. 1982; 127: 1233-7.

8. Summerhayes IC, Franks LM: Effects of donor age on neoplastic transformation of adult mouse bladder epithelium in vitro. J Natl Cancer Inst. 1979; 62: 1017-23.

9. Wang H, Satoh M, Abe H, Sunamura M, Moriya T, Ishidoya $\mathrm{S}$, et al.: Oncolytic viral therapy by bladder instillation using an E1A, E1B double-restricted adenovirus in an orthotopic bladder cancer model. Urology. 2006; 68: 674-81.

10. Soloway MS, Masters S: Urothelial susceptibility to tumor cell implantation: influence of cauterization. Cancer. 1980; 46: 1158-63.

11. Fodor I, Timiryasova T, Denes B, Yoshida J, Ruckle $\mathrm{H}$, Lilly M: Vaccinia virus mediated p53 gene therapy for bladder cancer in an orthotopic murine model. $\mathrm{J}$ Urol. 2005; 173: 604-9.

12. Luo Y, Chen X, O'donnell MA: Use of prostate specific antigen to measure bladder tumor growth in a mouse orthotopic model. J Urol. 2004; 172: 241420.

13. Debus E, Moll R, Franke WW, Weber K, Osborn M: Immunohistochemical distinction of human carcinomas by cytokeratin typing with monoclonal antibodies. Am J Pathol. 1984; 114: 121-30.

14. Cid Mouteira P, Ortíz Rey JA, Antón Badiola I, San Miguel Fraile P, Alvarez Alvarez C, et al.: Coordinated expression of cytokeratin 7 and 20 in transitional carcinoma of the bladder: diagnostic usefulness. Actas Urol Esp. 2002; 26: 279-84.

15. Schrier BP, Vriesema JL, Witjes JA, Kiemeney LA, Schalken JA: The predictive value of $\mathrm{p} 53$, p27(kip1), and alpha-catenin for progression in superficial bladder carcinoma. Eur Urol. 2006; 50: 76-82.

16. McKenney JK, Amin MB: The role of immunohistochemistry in the diagnosis of urinary bladder neoplasms. Semin Diagn Pathol. 2005; 22: 69-87.

17. Moltedo B, Faunes F, Haussmann D, De Ioannes P, De Ioannes AE, Puente J, et al.: Immunotherapeutic effect of Concholepas hemocyanin in the murine bladder cancer model: evidence for conserved antitumor properties among hemocyanins. J Urol. 2006; 176: 2690-5. 
18. Loskog AS, Fransson ME, Totterman TT: AdCD40L gene therapy counteracts $\mathrm{T}$ regulatory cells and cures aggressive tumors in an orthotopic bladder cancer model. Clin Cancer Res. 2005; 11: 8816-21.
Accepted after revision

February 22, 2008

\section{Correspondence address:}

Dr. Miguel Srougi

Rua Peixoto Gomide, 2055 / 81

São Paulo, SP, 01409-003, Brazil

Fax: + $55113257-8002$

E-mail: srougi@uol.com.br

\section{EDITORIAL COMMENT}

The study presented by Chade et al. shows a nice modification of the previously published orthotopic MB-49 bladder tumor model.

The main difference to the model optimization published by Günther et al. (1999) is the initial bladder lesion before tumor cell instillation. In the previous model by Günther et al. mice were placed with their backs on the ground plate of the cautery unit. To optimize contact, electrocardiogram electrode contact gel was used. The soft-tipped end of a springwire guide of a 24-gauge central venous catheter was inserted into the bladder via a Teflon catheter and gently pushed forward until it reached the bladder wall. The guide wire was attached to a cautery unit and a monopolar coagulation was applied for $5 \mathrm{~s}$ at the lowest setting $(5 \mathrm{~W})$. After removal of the guide wire, $0.05 \mathrm{~mL}$ of the tumor cell suspension was instilled. Chade et al. induced instead of physical alteration of the bladder wall a chemical lesion with intravesical silver nitrate.

Furthermore, in the original description catheters were after tumor cell instillation pinched off with a clamp, kept locked with a Luer-Lock closing cone, and left in place until the mice awakened. Using this method, a dwell time of approximately $3 \mathrm{~h}$. was given.

Here, a dwell time of 2 hours was performed by temporary stitches (presumably of the urethra).

The effectivity of the described technique is comparable to the previous model. The tumor take was almost $100 \%$ and all animals developed gross hematuria. However, the number of animals with pulmonary metastases, which was $20-70 \%$ before, was not mentioned here.

Chade et al. examined the Her2/neu, p53, CK7, and CEA expression in this model. Interestingly, p53 and Her2/neu staining was negative. One has to be aware that this can also be due to antibody-related problems since immunohistochemistry staining procedures are frequently more difficult in mouse tissue than in human tissue. We have previously examined Ki-67 (TEC-3) expression and found in the tumors up to $70 \%$ positive cells (unpublished data).

In general, the molecular comparison between human bladder cancers and mouse models is very interesting since the results of therapeutic applications 
may be easier to interpret. As the authors point out further molecular evaluations are planned. It would be highly interesting to perform these evaluations also in an humanized immune incompetent (SCID or Nude) mouse model. However, we were until now not able, to transfer the intravesical model into SCID mice since most mice died after bladder wall coagulation. Maybe the technical modification of Chade et al. would lead to better results.

\section{Dr. Ingo Kausch \\ Department of Urology University of Lubeck Medical School Lubeck, Germany E-mail:IngoKausch@uk-sh.de}

\section{EDITORIAL COMMENT}

Bladder cancer is well suited for experimental therapies due to the isolated bladder cavity in which therapies can be given locally. By transurethral noninvasive surgery, bladder tumors can easily be monitored and biopsies taken for further analyses. These features are beginning to attract a number of drug developers within a variety of fields including chemo-, immunoand gene therapy. Bladder cancer is one of few cancers that have excellent orthotopic murine experimental models that are closely mimicking the clinical situation $(1,2)$. Hence, in these orthotopic models, tumor biology can be studied and tumor therapy can be given locally by instillation via cauterization of urethra just as in the patients. In the current issue, Chade et al. are describing a novel system to enhance tumor take in experimental bladder cancer using silver nitrate $\left(\mathrm{AgNO}_{3}\right)$ as well as giving further insights into the biology of murine bladder tumors by performing a histopathological evaluation.

The most common murine bladder cancer cell lines are the mouse bladder-49 (MB49) and the mouse bladder tumor-2 (MBT2) cells (1-3). These two cell lines can be used in syngeneic C57BL6 and $\mathrm{C} 3 \mathrm{H}$ mice, respectively. The cell lines are utilized to create subcutaneous, orthotopic or metastatic tumors. In one of the first orthotopic models electrocautherization was used for tumor take. The electric pulse created a burn wound to which MB49 cells attached and formed tumors. In this model, the effect of Bacillus Calmette-
Guérin (BCG) therapy has been extensively evaluated and immunological mechanisms found in this model have later been proven transferable to human systems $(1,4)$. However, the electrocautherization model has a few drawbacks. The main issue is that it can be difficult to obtain the technical equipment necessary. Further, this model does not give 100\% tumor take which increases the number of mice needed per treatment group and may mask the true result in some treatment groups where differences are slim. Many groups have tried to obtain similar or better tumor take by chemical pretreatment of the bladder surface prior instillation of tumor cells. Agents tested are for example ethanol and poly-L-lysine (PLL) (2). Ethanol functions as an irritant and theoretically removes the mucin layer in the bladder thereby facilitating tumor take. The latter, PLL, is a polycathion that is thought to by its positive electrical charge become a bridge between the negatively charged urothelium and the tumor cells thereby aiding tumor attachment. PLL has so far been the only agent that repeatedly gives tumor take in all mice. In this issue, Chade et al has further improved the management of the orthotopic model via the use of $\mathrm{AgNO}_{3}$ pretreatment of the bladders to irritate the bladder wall prior tumor cell instillation. The tumor take is similar to that of PLL but the model as such saves time since this agent only needs a few seconds of incubation compared to PLL that needs to be incubated in the bladder for 10-20 min. prior tumor 
instillation. It will be of interest to evaluate effects of different therapies in this improved model.

The MB49 tumor cells have many similarities to its human equivalent in terms of antigens and immune escape mechanisms. The latter include infiltration of $\mathrm{T}$ regulatory cells in the growing tumor, expression of TGF, attraction of IL10-producing suppressor cells other than T regulatory cells etc (5-7). This makes the MB49 model excellent for evaluation of novel immunotherapies. The MB49 cells have expression of the male antigen $\mathrm{HY}$ and this antigen has been used as a pseudo tumor antigen when the cells are used in female mice (8). However, when antigen-directed approaches are evaluated, true tumor antigens need to be targeted. Carcinoembryonic antigen (CEA) is one of the first identified tumor antigens. It is expressed in about half of all human tumors, especially in adenocarcinomas $(9,10)$. The results from Chade et al demonstrate that MB49 cells express CEA as do human bladder cancer. This antigen is often used in tumor immunotherapy and the MB49 model can, hence, serve as a model system not only for bladder cancer but for all CEA positive tumors.

The murine experimental MB49 model gives new insights to tumor progression, survival and immune escape in human bladder cancer. Currently, there are several novel therapies such as immune and gene therapy that are proven potent in the MB49 model and now translated into clinical Phase I and II trials. It is important to further investigate murine experimental models to simplify the techniques as well as to further enlighten biological phenomena that may be translated into human cancer and get us closer to better and more refined drugs.

\section{REFERENCES}

1. Günther JH, Jurczok A, Wulf T, Brandau S, Deinert I, Jocham D, et al.: Optimizing syngeneic orthotopic murine bladder cancer (MB49). Cancer Res. 1999; 59: 2834-7.

2. Loskog A, Ninalga $C$, Hedlund T, Alimohammadi M, Malmström PU, Tötterman TH: Optimization of the MB49 mouse bladder cancer model for adenoviral gene therapy. Lab Anim. 2005; 39: 384-93.

3. Torti SV, Golden-Fleet M, Willingham MC, Ma R, Cline M, Sakimoto Y, et al.: Use of green fluorescent protein to measure tumor growth in an implanted bladder tumor model. J Urol. 2002; 167: 724-8.

4. Suttmann H, Riemensberger J, Bentien G, Schmaltz D, Stöckle M, Jocham D, et al.: Neutrophil granulocytes are required for effective Bacillus CalmetteGuérin immunotherapy of bladder cancer and orchestrate local immune responses. Cancer Res. 2006; 66: 8250-7.

5. Loskog A, Dzojic H, Vikman S, Ninalga C, Essand M, Korsgren O, et al.: Adenovirus CD40 ligand gene therapy counteracts immune escape mechanisms in the tumor Microenvironment. J Immunol. 2004; 172: 7200-5.

6. Loskog AS, Fransson ME, Totterman TT: AdCD40L gene therapy counteracts $T$ regulatory cells and cures aggressive tumors in an orthotopic bladder cancer model. Clin Cancer Res. 2005; 11: 8816-21.

7. Loskog A, Ninalga C, Paul-Wetterberg G, de la Torre M, Malmström PU, Tötterman TH: Human bladder carcinoma is dominated by T-regulatory cells and Th1 inhibitory cytokines. J Urol. 2007; 177: 353-8.

8. Melchionda F, McKirdy MK, Medeiros F, Fry TJ, Mackall CL: Escape from immune surveillance does not result in tolerance to tumor-associated antigens. J Immunother. 2004; 27: 329-38.

9. Huang EH, Kaufman HL: CEA-based vaccines. Expert Rev Vaccines. 2002; 1: 49-63.

10. Hörig H, Medina FA, Conkright WA, Kaufman HL: Strategies for cancer therapy using carcinoembryonic antigen vaccines. Expert Rev Mol Med. 2000; 2: 124.
Dr. Angelica Loskog, PhD Clinical Immunology Division Rudbeck Laboratory, Uppsala University Uppsala, Sweden

Email: angelica.loskog@klinimm.uu.se 


\section{EDITORIAL COMMENT}

An animal model that closely resembles human bladder cancer is needed for preclinical studies on the pathogenesis of bladder cancer and the development of therapeutic strategies for treating this disease. This paper describes a syngeneic murine bladder tumor model that is developed by intravesical implantation of MB49 cells, a commonly used murine bladder cancer cell line of the C57BL/6 origin. The authors have established the experimental conditions that result in a high incidence of orthotopic tumor in mice $(96.7 \% ; 29$ out of 30 mice on day 15). By using a small volume of silver nitrate to traumatize the urothelium, the authors have demonstrated the feasibility of this method for intravesical bladder tumor implantation.
The implanted animals developed a solid tumor inside the bladder that mimics human urothelial invasive carcinoma in histopathology. Immunohistochemical analysis showed the strong expression of cytokeratin 7 and carcinoembrionic antigen on the surface of MB49 tumor cells, which is similar to human urothelial cancer. These surface markers facilitate the identification of primary tumor when metastasis occurs. Although this model is feasible and provides a high rate of tumor intake, this model needs to be improved for its variability in tumor growth. Nevertheless, this model provides a useful means for the therapeutic studies of bladder cancer including immunotherapy, chemotherapy, and gene therapy.

Dr. Yi Luo

Department of Urology University of Iowa

Roy J. and Lucille A. Carver College of Medicine Iowa City, Iowa, USA E-mail:yi-luo@uiowa.edu 